OPEN ACCESS

Edited by:

loan Opris,

University of Miami, United States

Reviewed by:

Shane Matsune Fresnoza,

University of Graz, Austria

José M. Delgado-García,

Universidad Pablo de Olavide, Spain

*Correspondence:

Adam Kirton

adam.kirton@ahs.ca

tThese authors have contributed equally to this work and are co-first

authors

Specialty section:

This article was submitted to

Neural Technology,

a section of the journal

Frontiers in Neuroscience

Received: 17 August 2018 Accepted: 11 October 2018

Published: 31 October 2018

Citation:

Cole L, Giuffre A, Ciechanski P, Carlson HL, Zewdie E, Kuo H-C and Kirton A (2018) Effects of High-Definition and Conventional

Transcranial Direct-Current

Stimulation on Motor Learning in Children. Front. Neurosci. 12:787.

doi: 10.3389/fnins.2018.00787

\section{Effects of High-Definition and Conventional Transcranial Direct-Current Stimulation on Motor Learning in Children}

\author{
Lauran Cole ${ }^{1,2,3+}$, Adrianna Giuffre ${ }^{1,3 t}$, Patrick Ciechanski ${ }^{1}$, Helen L. Carlson ${ }^{4,5}$, \\ Ephrem Zewdie ${ }^{2,4}$, Hsing-Ching Kuo ${ }^{4,5}$ and Adam Kirton ${ }^{2,3,4,5,6 *}$ \\ ${ }^{1}$ Department of Neurosciences, University of Calgary, Calgary, AB, Canada, ${ }^{2}$ Cumming School of Medicine, University \\ of Calgary, Calgary, AB, Canada, ${ }^{3}$ Hotchkiss Brain Institute, University of Calgary, Calgary, AB, Canada, ${ }^{4}$ Department \\ of Pediatrics, University of Calgary, Calgary, AB, Canada, ${ }^{5}$ Alberta Children's Hospital Research Institute, University \\ of Calgary, Calgary, AB, Canada, ${ }^{6}$ Department of Clinical Neurosciences, University of Calgary, Calgary, AB, Canada
}

Background: Transcranial direct current stimulation (tDCS) can improve motor learning in children. High-definition approaches (HD-tDCS) have not been examined in children.

Objectives/Hypothesis: We hypothesized that primary motor cortex HD-tDCS would enhance motor learning but be inferior to tDCS in children.

Methods: Twenty-four children were recruited for a randomized, sham-controlled, double-blinded interventional trial (NCT03193580, clinicaltrials.gov/ct2/show/NCT03193580) to receive (1) right hemisphere (contralateral) primary motor cortex (M1) $1 \mathrm{~mA}$ anodal conventional $1 \times 1 \mathrm{tDCS}$ (tDCS), (2) right M1 $1 \mathrm{~mA}$ anodal $4 \times 1 \mathrm{HD}$-tDCS (HD-tDCS), or (3) sham. Over five consecutive days, participants trained their left hand using the Purdue Pegboard Test $\left(\mathrm{PPT}_{\mathrm{L}}\right)$. The Jebsen-Taylor Test, Serial Reaction Time Task, and right hand and bimanual PPT were also tested at baseline, post-training, and 6-week retention time (RT).

Results: Both the tDCS and HD-tDCS groups demonstrated enhanced motor learning compared to sham with effects maintained at 6 weeks. Effect sizes were moderate-tolarge for tDCS and HD-tDCS groups at the end of day 4 (Cohen's $d$ tDCS $=0.960$, HD-tDCS $=0.766)$ and day 5 (tDCS $=0.655$, HD-tDCS $=0.851)$. Enhanced motor learning effects were also seen in the untrained hand. HD-tDCS was well tolerated and safe with no adverse effects.

Conclusion: HD-tDCS and tDCS can enhance motor learning in children. Further exploration is indicated to advance rehabilitation therapies for children with motor disabilities such as cerebral palsy.

Clinical Trial Registration: clinicaltrials.gov, identifier NCT03193580.

Keywords: tDCS, HD-tDCS, motor learning, non-invasive brain stimulation, developmental neuroplasticity, child

Abbreviations: AMPED, accelerated motor learning in pediatrics; ANOVA, analysis of variance; BDNF, brain-derived neurotrophic factor; CP, cerebral palsy; HD-tDCS, high-definition transcranial direct current stimulation; JTT, Jebsen-Taylor Test of Hand Function; LTP, long term potentiation; M1, primary motor cortex; PPT, Purdue Pegboard Test; REDCap, Research Electronic Data Capture; SRTT, Serial Reaction Time Task; tDCS, transcranial direct current stimulation; TMS, transcranial magnetic stimulation. 


\section{INTRODUCTION}

Transcranial direct stimulation (tDCS), a form of non-invasive brain stimulation, has potential to modulate cortical excitability, human brain function, and behavior. Such promise has advanced studies across diverse brain disorders with over 33,000 sessions completed (Bikson et al., 2016). Safety and tolerability are well defined but mechanisms are poorly understood. Both preclinical and human evidence suggests long-term potentiation (LTP)-like mechanisms are involved (Stagg and Nitsche, 2011). As is often the case with emerging therapeutics, pediatric populations have been understudied in tDCS research where $<2 \%$ of studies have been dedicated to the developing brain.

The primary motor cortex (M1), a key structure in motor skill learning, can be purposefully modulated with brain stimulation (Reis et al., 2008). tDCS animal models have demonstrated how polarizing, subthreshold direct currents can alter cortical excitability, neuronal firing and the size of evoked potentials (Bindman et al., 1964). tDCS over M1 increases cortical excitability (Nitsche and Paulus, 2000) and, when paired with training of the contralateral hand, improves motor performance within single (Nitsche et al., 2003; Boggio et al., 2006; Vines et al., 2006) and multiple (Reis et al., 2009) sessions. Cathodal stimulation of the ipsilateral M1 can also improve motor skill acquisition, presumably via effects on transcallosal, interhemispheric motor networks (Fregni and Pascual-Leone, 2007). Recently, we demonstrated that such M1 tDCS approaches can enhance motor learning in healthy children over 3 days of training with retained effects and large effect sizes (Ciechanski and Kirton, 2016). Stimulation was well-tolerated with no adverse events. Limited evidence suggests tDCS- induced electric fields differ in the pediatric brain though mechanistic investigations of tDCS in pediatrics are lacking (Stagg and Nitsche, 2011; Dayan et al., 2013; Kessler et al., 2013; Moliadze et al., 2015).

High-definition tDCS (HD-tDCS) may provide more focal current delivery to better target functional cortical regions. By placing a central anode surrounded by four cathodes, $4 \times 1 \mathrm{HD}$ tDCS can be applied in a more focused manner with generation of stronger regional electric fields (Dmochowski et al., 2011). HD-tDCS can increase motor adaptation within a single session (Doppelmayr et al., 2016), and bimanual hand dexterity over multiple days in adults (Pixa et al., 2017). To date, HD-tDCS has not been examined in a pediatric population.

The ease of application of tDCS has promoted its early translation toward childhood disability and CP. Perinatal stroke is the leading cause of hemiparetic $\mathrm{CP}$, affecting millions worldwide with few effective treatments (Oskoui et al., 2013; Kang et al., 2016). Perinatal stroke is an ideal human model of developmental plasticity where targeting M1 has shown potential for therapeutic neuromodulation (Kirton, 2016). Although the models are different, trials in adult stroke hemiparesis suggest tDCS may facilitate motor rehabilitation (Kang et al., 2016; Elsner et al., 2017). Preliminary evidence of efficacy has been suggested in trials of contralesional tDCS in hemiparetic children (Kirton et al., 2017; Gillick et al., 2018). There is a pressing need to optimize tDCS enhancement of motor learning in pediatrics to advance such therapies and better outcomes for disabled children.
We therefore conducted a sham-controlled, double-blind, randomized trial to compare the effects of M1 tDCS and HDtDCS on motor learning in typically developing children.

\section{MATERIALS AND METHODS}

\section{Trial Design and Participants}

Accelerated Motor Learning in Pediatrics (AMPED) was a randomized, double-blind, single-center, sham-controlled interventional trial. The study was registered at clinicaltrials.gov (NCT03193580). This study was carried out in accordance with the recommendations of the University of Calgary Research Ethics Board with informed written consent from all participants. All participants and their guardians provided written informed consent and assent when applicable in accordance with the Declaration of Helsinki. The protocol was approved by the Conjoint Health Research Ethics Board, University of Calgary (REB16-2474).

Participants were recruited through community and school outreach programs and the Healthy Infants and Children Clinical Research Program. Inclusion criteria were: (1) age 12-18 years, (2) right handed (self/parent report and Edinburgh Handedness Inventory), (3) typical neurodevelopment, (4) no major medical conditions, and (5) informed consent/assent. Persons with neuropsychiatric diagnoses/medication or implanted devices were excluded.

Each participant underwent the same testing, training, and stimulation procedures over five consecutive days. The complete study design and flow is shown in Figure 1.

Previous evidence of tDCS-enhanced motor learning in children (Ciechanski and Kirton, 2016) suggested moderate-tolarge effect sizes and typical means and variances (1.5- to 2 -fold increases with mean standard deviation of 0.71 ). Combining these with $\alpha=0.05$ and a power of $85 \%$ estimated a total requirement of 24 participants (8 per stimulation group) using an online sample size calculator ${ }^{1}$.

\section{Randomization, Blinding, and Concealment}

Using the Research Electronic Data Capture tool (REDCap), participants were randomized into three groups (1:1:1): (1) right M1 $1 \mathrm{~mA}$ anodal conventional $1 \times 1 \mathrm{tDCS}$ (tDCS), (2) right M1 $1 \mathrm{~mA}$ anodal $4 \times 1 \mathrm{HD}$-tDCS (HD-tDCS), or (3) sham. Participants and their parents were blinded to treatment assignment consistent with previous pediatric trials (Ciechanski and Kirton, 2016). A post-interventional questionnaire asked participants to guess which intervention they received and why. Only the investigator administering the stimulation was aware of the treatment group.

\section{Motor Learning Measures}

The primary motor learning measure was the PPT, a validated measure of hand dexterity commonly used in motor learning studies (Tiffin and Asher, 1948; Gardner and Broman, 1979).

\footnotetext{
${ }^{1}$ https://www.stat.ubc.ca/ rollin/stats/ssize/n2.html
} 


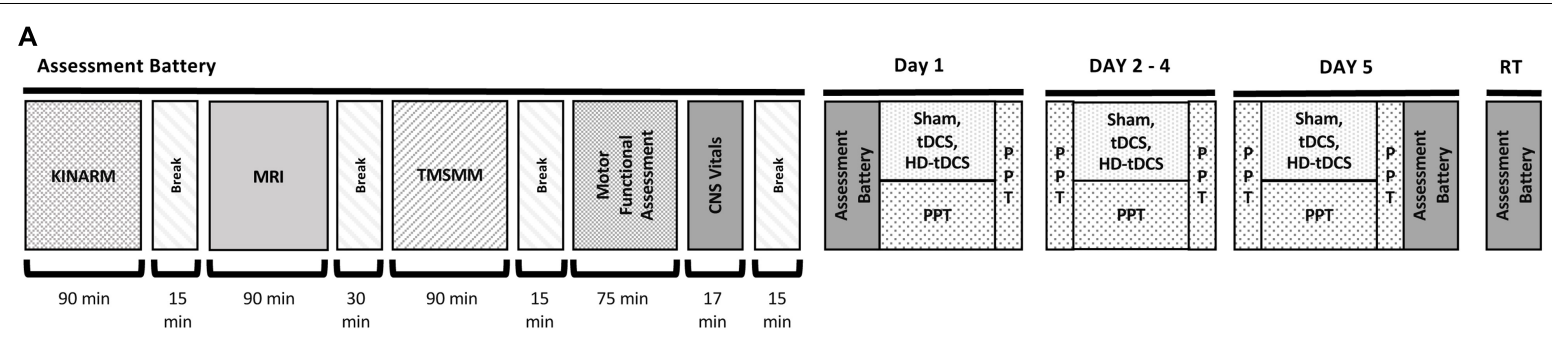

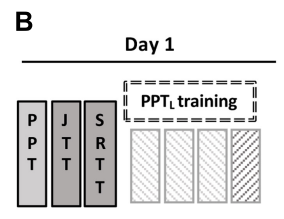

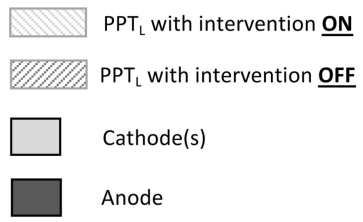

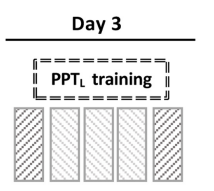
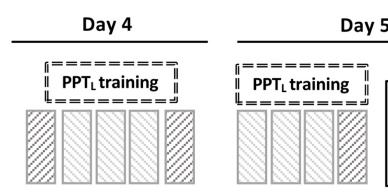

Day 5

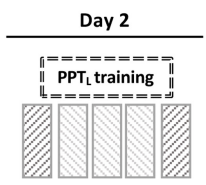

C

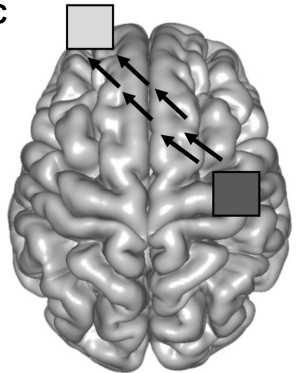

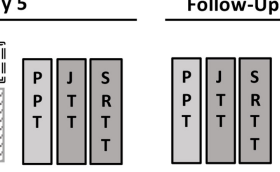

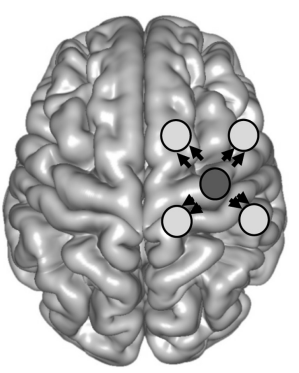

FIGURE 1 | Accelerated motor learning in pediatrics (AMPED) protocol. (A) Participants received an MRI, complete tasks in a virtual reality KINARM robotic system, received TMS motor mapping (TMSMM), completed a series of motor assessments and then received training paired with non-invasive brain stimulation interventions. On days 2-4, subjects perform the PPT during intervention. Participants repeat the Day 1 tasks on Day 5 (with training) and at a 6 -weeks retention testing follow up (RT). (B) PPT training is paired with stimulation by treatment groups with electrode montages (C) shown for tDCS (left) and HD-tDCS (right) where dark gray electrodes are anodes and light gray electrodes are cathodes. Black arrows represent the direction of current flow from anode to cathode(s).

The PPT consists of four subtests, the first three require participants to place as many pegs as they can in a pegboard in $30 \mathrm{~s}$ with their left hand $\left(\mathrm{PPT}_{\mathrm{L}}\right)$, right hand $\left(\mathrm{PPT}_{\mathrm{R}}\right)$, and bimanually $\left(\mathrm{PPT}_{\mathrm{LR}}\right)$. The sum of the three scores was also generated $\left(\mathrm{PPT}_{\mathrm{S}}\right)$. The final subtest was a bimanual assembly task $\left(\mathrm{PPT}_{\mathrm{A}}\right)$. The total number of pegs was recorded. The $\mathrm{PPT}_{\mathrm{L}}$ was used to train and measure motor learning as a challenging task for children to learn without reaching a skill "ceiling" effect. The $\mathrm{PPT}_{\mathrm{R}}$ evaluated effects in the untrained hand. Secondary, untrained motor outcomes included the JTT which assessed six subtests of common unimanual hand functions (Elizabeth Reedman et al., 2015) and the SRTT which measured reaction time and implicit motor learning (Nissen and Bullemer, 1987; Honda et al., 1998).

\section{Intervention: tDCS and HD-tDCS}

Participants received direct-current stimulation or sham during each training session using a conventional $1 \times 1$ tDCS or a $4 \times 1$ HD-tDCS system (Soterix Medical Inc., New York). Using the T1 images acquired from their MRI, each participant's right M1 was localized using neuronavigation via an optical detection camera system (Brainsight2, Rouge Research Inc., Montreal; Polaris NDI Medical Solutions, Ontario, Canada). Robotic single-pulse TMS localized the "hotspot" for the first dorsal interosseous muscle of the left hand using established criteria (Zewdie and Kirton, 2016).
For tDCS and sham, two saline-soaked sponge electrodes (25 $\mathrm{cm}^{2}$, SNAPpad, Soterix) were applied to the scalp. The active electrode (anode) centered over the right M1 and the cathode over the contralateral supraorbital area (Figure 1C). Electrodes were held in place with a light plastic pediatric "headband" (SNAPstrap, Soterix Medical Inc., New York). The electrodes were then connected to a $1 \times 1$ DC SMARTscan Stimulator (Soterix). This was the same montage described in both adult and pediatric tDCS motor learning studies (Vines et al., 2006; Reis et al., 2009; Reis and Fritsch, 2011; Ciechanski and Kirton, 2016).

For HD-tDCS, the montage targeted the right M1 as described elsewhere (Caparelli-Daquer et al., 2012; Villamar et al., 2013; Richardson et al., 2015). Participants wore "cap" with pre-existing electrode holes. The anode was centered over the right M1 with four cathodes spaced $\sim 5 \mathrm{~cm}$ away to establish a ring-like orientation (Figure 1C) (12 cm diameter, Sintered ring HDelectrode, Soterix) (Datta et al., 2009; Kuo et al., 2013; Villamar et al., 2013, p. 1; Alam et al., 2014). Electrodes were then connected to a $4 \times 1 \mathrm{HD}$-tDCS Adaptor and a SMARTscan Stimulator (Soterix).

During active stimulation, current was initially ramped up to $1 \mathrm{~mA}$ over $30 \mathrm{~s}$ and maintained for $20 \mathrm{~min}$ with automatic continuous current-control. Current was then ramped down to $0 \mathrm{~mA}$ over $30 \mathrm{~s}$ and was current-controlled based on continuous sampling of resistance. In the sham group, current was initially ramped to $1 \mathrm{~mA}$ over $30 \mathrm{~s}$ then immediately ramped down to 
$0 \mathrm{~mA}$. During the final $30 \mathrm{~s}$, the current ramped up to $1 \mathrm{~mA}$ and back to $0 \mathrm{~mA}$. This sham procedure has been validated in subjects naïve to tDCS (Ambrus et al., 2012).

\section{Training Protocol}

The sequence of motor measures, motor training, and stimulation are summarized in Figure 1B. Participants could complete all tasks within a single session on Day $1(\sim 8 \mathrm{~h})$ or split up Day 1 into two consecutive days ( $\sim 4$ h each). On Day 1, baseline skill was measured by completing all motor tasks. Each participant then trained the $\mathrm{PPT}_{\mathrm{L}}$ while receiving either tDCS, HD-tDCS, or sham. Training occurred over 20 min of stimulation, consisting of three trials per epoch performed at minute 5, 10, and 15. After stimulation, the electrodes were removed and participants completed a safety and tolerability questionnaire (below). The $\mathrm{PPT}_{\mathrm{L}}$ was then performed again. On days 2, 3, and 4, participants performed the same $\mathrm{PPT}_{\mathrm{L}}$ sequence, beginning with a baseline test followed by the same 20-min training protocol during stimulation. On Day 5, participants repeated all assessments performed on Day 1, starting with the same training protocol. Participants returned $6 \pm 1$ weeks later and performed the same order of assessments as Day 1 .

Each assessment was video-taped and re-scored by a blinded team member for quality assurance. Learning curves generated for the $\mathrm{PPT}_{\mathrm{L}}$ compared the score difference at each training point with the baseline score. Skill decay was measured by comparing the 6-week follow-up score with the Day 5 post-training score. Online effects (within-day training) were determined by comparing baseline and final scores of each day. Offline effects (consolidation) were measured by comparing baseline scores each day to the final score from the previous day.

\section{Safety and Tolerability}

Participants completed a pediatric non-invasive brain stimulation safety and tolerability questionnaire (Garvey et al., 2001) immediately following each session (days 1-5). The duration and severity of any symptoms were reported including headache, neck pain, unpleasant tingling, itching, burning, fatigue, nausea, and lightheadedness. Participants were asked to rank the tolerability of their session compared to seven common childhood experiences. As the first study of HD-tDCS in children, a neuropsychological battery was completed at baseline and following the final stimulation session. A validated, computerized assessment (CNS Vital Signs) evaluated neurocognitive status (Gualtieri and Johnson, 2006). An interim safety analysis was conducted by two blinded researchers after the first eight subjects to exclude any drop-in motor function (reduction of PPT) of either hand or serious adverse events.

\section{Statistical Analysis}

For the primary hypothesis, a linear mixed effect model analysis was employed (SPSS 25.0.0, IBM, Armonk, NY, United States) with fixed effects for Group, Day, and the interaction of Group and Day and random effects for Subjects including the intercept to account for repeated measures. The dependent variable for linear mixed modeling was PPTL score, independent variables being group and day. Analysis for secondary continuous outcomes (SigmaPlot 12.5, Systat Software Inc.; San Jose) included one-way analyses of variance (ANOVA) and Chisquare/Fisher's exact test for dichotomous variables to compare group demographics, baseline motor scores, and tolerability. Paired $t$-tests analyzed differences in left and right-hand motor scores, skill decay, and online/offline effects. Effect sizes were reported as Cohen's $d$. Repeated measures ANOVA was used to analyze changes in JTT and SRTT scores. Holm-Sidak post hoc corrections were performed to correct for multiple comparisons. To examine possible effects of baseline function, participants were divided into high and low performers based on baseline $\mathrm{PPT}_{\mathrm{L}}$ scores above or below the median.

\section{Replication}

To evaluate replicability of previous studies while adding to the power of the current study, we combined our data with that of a previous, similar trial (Ciechanski and Kirton, 2016). Both studies had the same inclusion criteria and applied right M1

TABLE 1 | Participant demographics and baseline motor function.

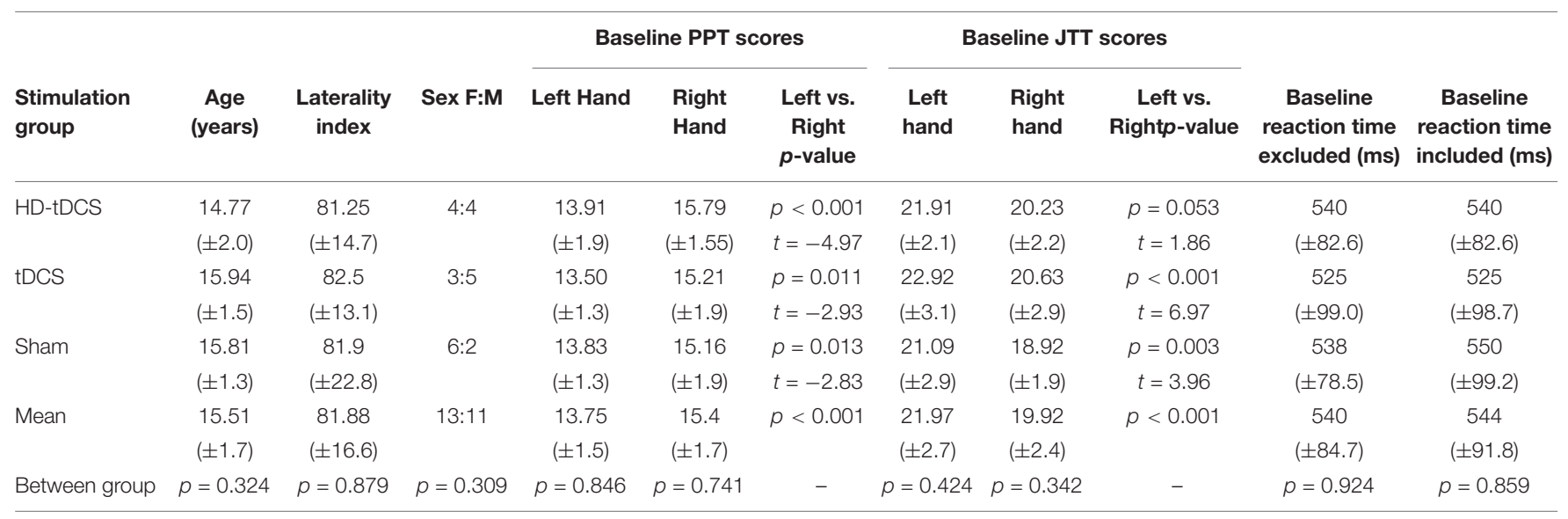

JTT, Jebsen-Taylor Test; PPT, Purdue Pegboard Test. 
tDCS $(1 \mathrm{~mA})$ or sham during 20 min of $\mathrm{PPT}_{\mathrm{L}}$ training over three consecutive days. Accordingly, learning curves over 3 days of training from sham controls and anodal tDCS groups were combined ( $n=14$ for both groups). The linear mixed modeling analysis was repeated with the combined data. The fixed effects were Group, Day, and the interaction of Group and Day. The random effects were Subjects including the intercept to account for repeated measures.

\section{RESULTS}

\section{Population Characteristics}

Twenty-four participants were recruited (median age 15.5 years, range $12-18,52 \%$ female). All completed all stages and outcomes

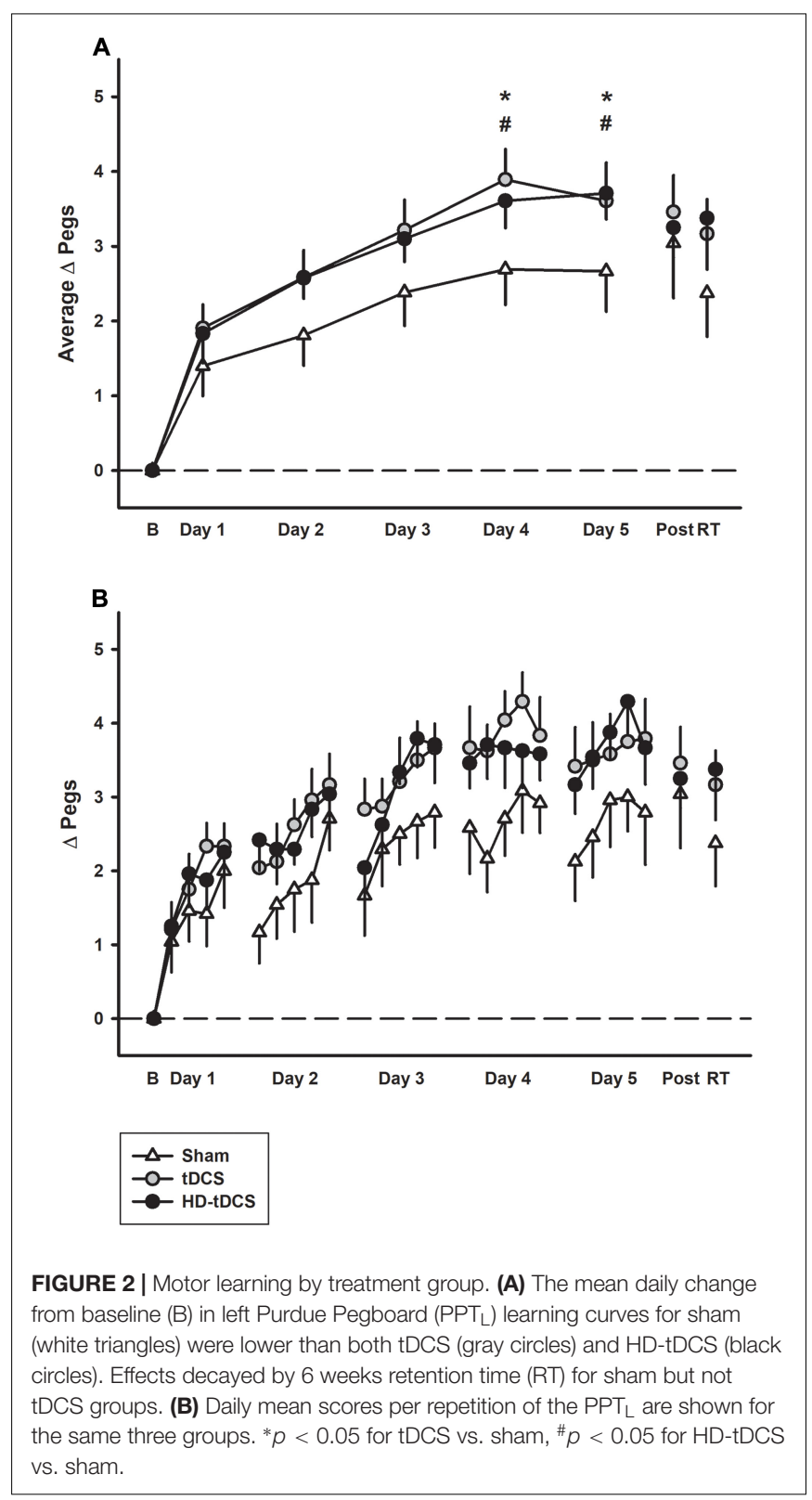

with no drop-outs. Demographics and baseline motor function across groups are shown in Table 1. Age, sex, handedness, and function did not differ between groups (all $p>0.3$ ). All groups demonstrated higher $\mathrm{PPT}_{\mathrm{R}}$ compared to $\mathrm{PPT}_{\mathrm{L}}$ scores $(p<0.001)$.

\section{Motor Learning}

Learning curves of similar morphology were generated across subjects and groups. Daily motor learning and daily average learning curves by group are shown in Figure 2. Regardless of intervention, participants demonstrated motor learning over the 5 days with the rate dissipating over time $[F(2,24)=23.7$, $p<0.001]$. Linear modeling demonstrated a significant interaction effect of day and intervention on the rate of learning. The active stimulation groups demonstrated enhanced learning (increase in pegs/day) compared to sham (tDCS $p=0.042$, HDtDCS $p=0.049)$. The sham group improved their $\mathrm{PPT}_{\mathrm{L}}$ score by $0.508 \pm 0.190$ pegs/day compared to $0.703 \pm 0.269$ for the tDCS group and $0.697 \pm 0.269$ for the HD-tDCS group. At both days 4 and 5, the tDCS and HD-tDCS groups had larger improvements in the daily average $\mathrm{PPT}_{\mathrm{L}}$ score compared to sham (tDCS: $p=0.026, p=0.038$; HD-tDCS: $p=0.043, p=0.05$; days 4 and 5 , respectively). Moderate to large effect sizes were observed in the tDCS and HD-tDCS groups at the end of day 4 (Cohen's $d$ tDCS $=0.960$, HD-tDCS $=0.766$ ) and day 5 (Cohen's $d \mathrm{tDCS}=0.655, \mathrm{HD}-\mathrm{tDCS}=0.851)$.

\section{Retention}

Learning effects were retained in the tDCS and HD-tDCS groups with no decrease in skill performance between end of training and the retention assessment. In contrast, skill decay was observed in the sham group $(p=0.034, t=2.16)$. At retention testing, there

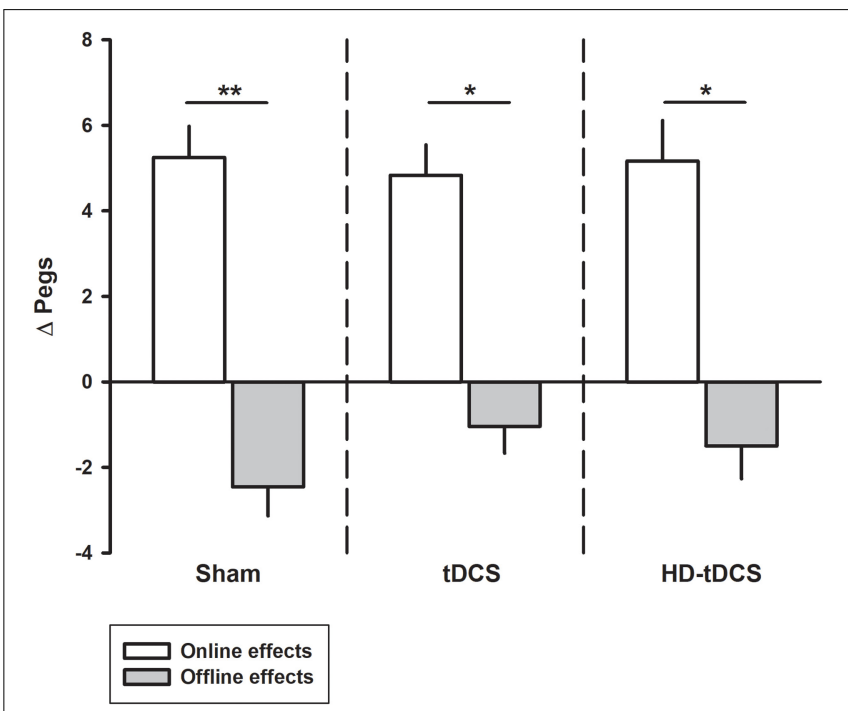

FIGURE 3 | Online and offline learning effects on left hand Purdue Pegboard $(\mathrm{PPT})$ for the three intervention groups. The online effects represent the difference in $P P T_{L}$ score from the first and last training point of the day. The offline learning represents the difference between the last training time point of the day to the first training point of the following day. ${ }^{*} p<0.05,{ }^{* *} p<0.01$. 
was no difference in $\mathrm{PPT}_{\mathrm{L}}$ scores between intervention groups $(p=0.456)$.

\section{Online/Offline Learning}

Performance improvements occurred primarily through online learning (all $p<0.003, t>4.03$; Figure 3). There were no differences seen between tDCS or HD-tDCS and sham in the amount of online learning. Offline effects contributed to a significant loss of skill in the sham and HD-tDCS group (sham $p=0.004, t=3.64$; HD-tDCS $p=0.046, t=1.95)$ but not the tDCS group $(p=0.070, t=1.67)$.

\section{Low Versus High Performers}

The median baseline $\mathrm{PPT}_{\mathrm{L}}$ score for all participants was 13.33 with 11 participants above this classified as high performers and the remaining participants being low performers (Figure 4). In the low performance group, the tDCS and HD-tDCS groups demonstrated greater improvements compared to sham. The high performer group did not show any difference in learning across groups.

\section{Secondary, Untrained Motor Outcomes}

The effects of intervention group on the secondary motor outcomes are shown in Figure 5. $\mathrm{PPT}_{\mathrm{R}}$ scores increased following training in the tDCS and HD-tDCS groups (both $p<0.042$ ) but did not change in the sham group (Figure 5A, $p=0.076$ ). $\mathrm{PPT}_{\mathrm{R}}$ scores were not correlated with $\mathrm{PPT}_{\mathrm{L}}$ improvements $(r=0.266$, $p=0.208)$. There was no decay in $\mathrm{PPT}_{\mathrm{R}}$ scores at retention testing in all groups.

PPT $_{L R}$ scores improved in all groups: sham $(p=0.016$, $t=2.69), \operatorname{tDCS}(p=0.003, t=4.00)$, and HD-tDCS groups $(p<0.001, t=8.62)$ with no decay. There was no difference between the three groups at post-training $(p=0.664)$. $\mathrm{PPT}_{\mathrm{LR}}$ scores at post-training correlated with change in $\mathrm{PPT}_{\mathrm{L}}$ $(r=0.564, p=0.004)$. Regardless of group, participants showed improvements in $\mathrm{PPT}_{\mathrm{S}}$ scores (all $p<0.006$ ), again without decay. There was also an improvement in $\mathrm{PPT}_{\mathrm{A}}$ for all groups (all $p<0.03, t>2.53$ ) and no decay. There was no difference between the intervention groups for change in $\mathrm{PPT}_{\mathrm{A}}(p=0.506)$. There was an improvement in $\mathrm{PPT}_{\mathrm{A}}$ from post-training to retention in the tDCS group $(p=0.05)$. There was no correlation between $\mathrm{PPT}_{\mathrm{A}}$ and $\mathrm{PPT}_{\mathrm{L}}$ improvements $(r=-0.032, p=0.881)$.

Jebsen-Taylor Test performance is summarized in Figure 5B. All three treatment groups improved their $\mathrm{JTT}_{\mathrm{L}}$ scores from baseline to post-training $(p<0.003)$ and baseline to retention testing $(p<0.019)$. In the untrained hand, $\mathrm{JTT}_{\mathrm{R}}$ scores improved over time from baseline to post-training $(p=0.005)$ and from baseline to retention testing $(p=0.019)$. JTT $_{\mathrm{R}}$ scores significantly improved in the tDCS group from baseline to post-training $(p=0.016)$ and in the HD-tDCS group from baseline to retention

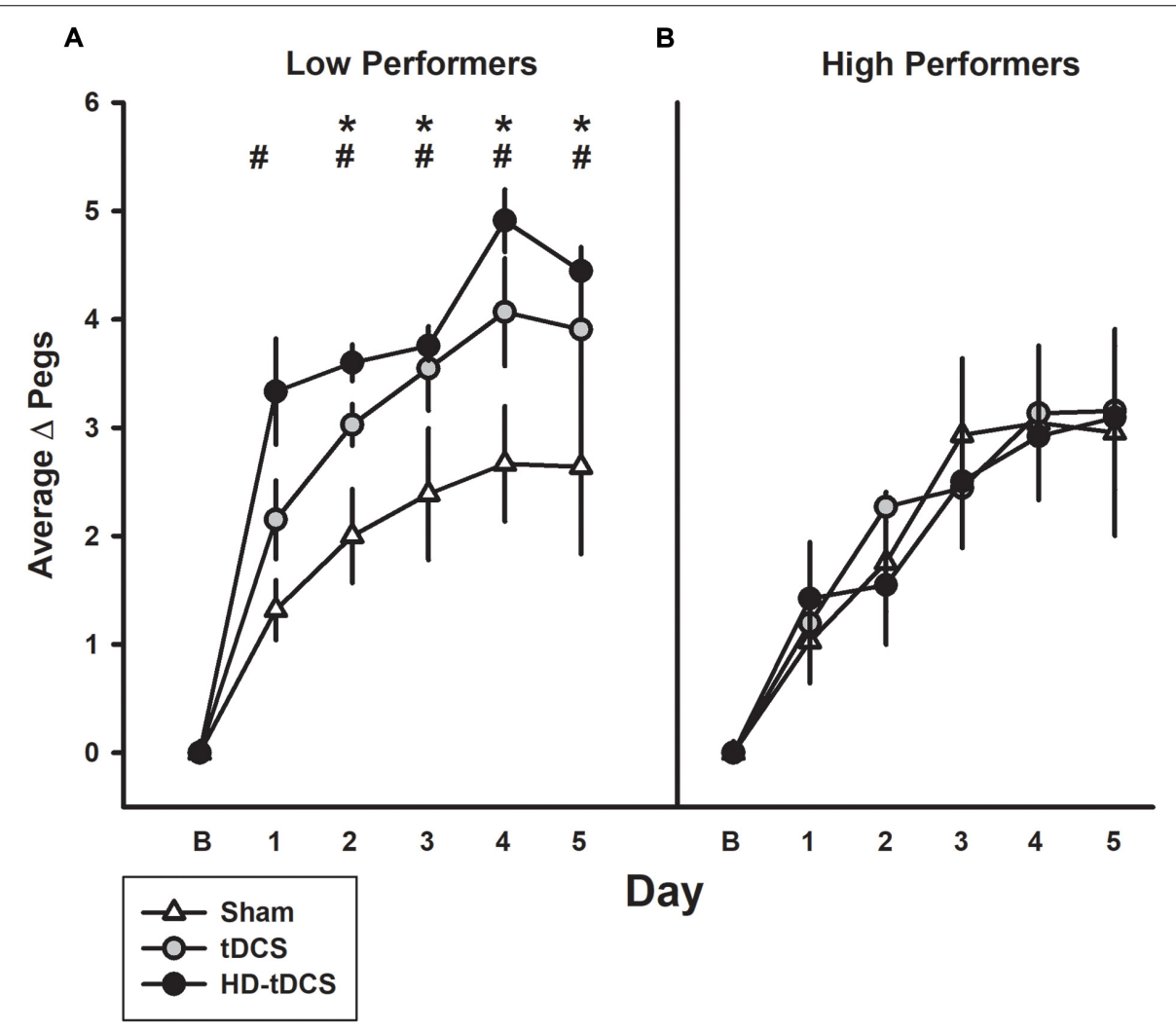

FIGURE 4 | Effect of performance status on motor learning enhancement. (A) Low performers (baseline PPT $L$ below the median score) demonstrated marked separation of PPT L learning curves with tDCS (gray circles) and HD-tDCS (black circles) outperforming sham (white triangles). (B) Treatment group effects were not observed for high performers. B refers to baseline. ${ }^{*} p<0.05$ for sham vs. tDCS, ${ }^{\#} p<0.05$ for sham vs. HD-tDCS. 

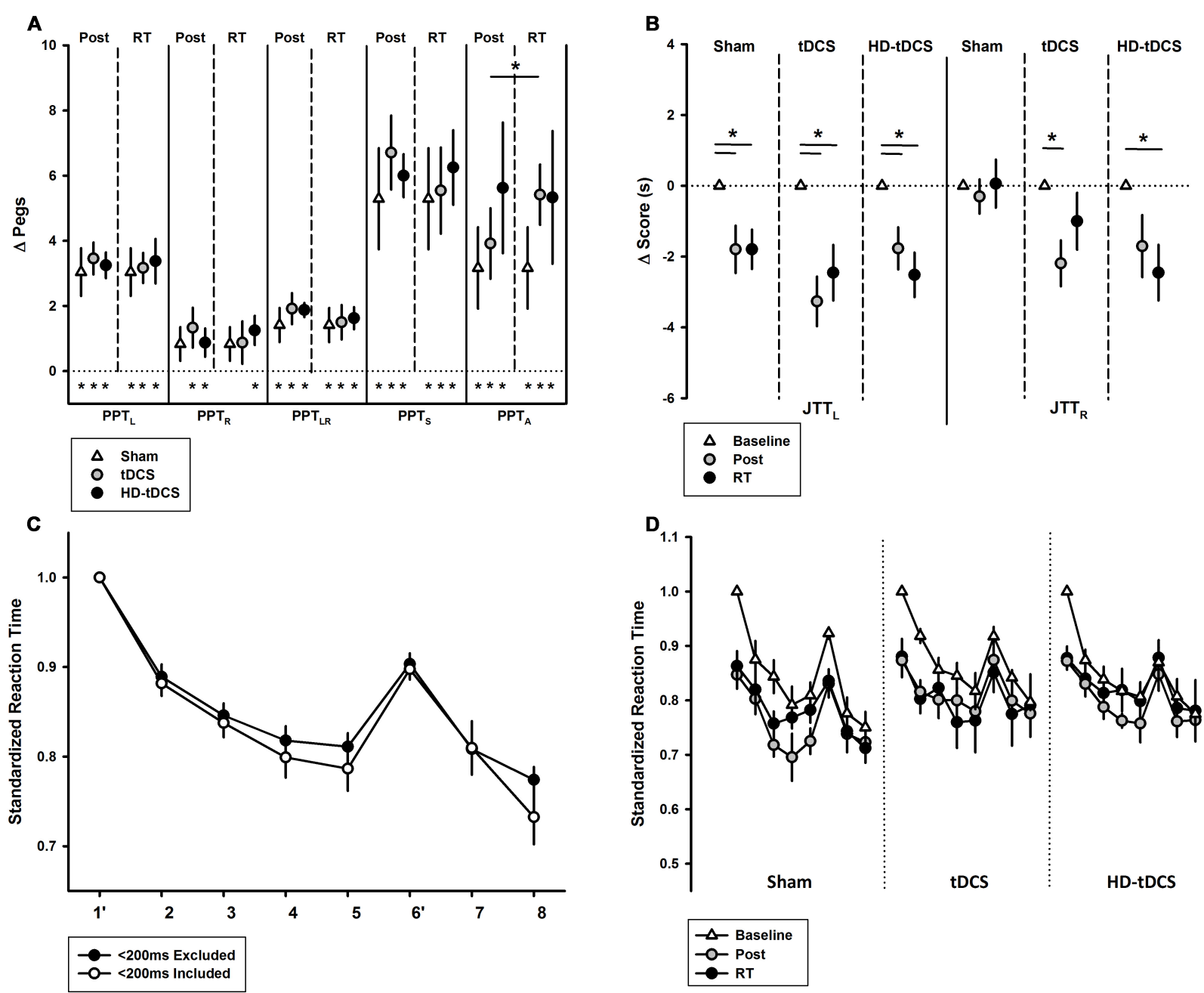

FIGURE 5 | Secondary motor outcomes. (A) Change in Purdue Pegboard Test (PPT) scores at post-training and retention time (RT) demonstrated treatment group effects for PPT $T_{A}$. PPT subtests are left (PPT $)$, right (PPT $)$, bimanual (PPT $\left.\mathrm{LR}_{\mathrm{R}}\right)$, sum of scores (PPT $)$, and assembly $\left(\mathrm{PPT}_{\mathrm{A}}\right) \cdot{ }^{*} p<0.05$. (B) Jebsen-Taylor Test of Hand Function left and right ( $\mathrm{JTT} L, \mathrm{JT} R$ ) demonstrated treatment group effects bilaterally at post-training and RT. (C) Serial Reaction Time Task (SRTT) curves with and without $<200 \mathrm{~ms}$ responses are shown. Blocks 1 and 6 are random while all others follow a 12-character sequence. (D) SRTT by intervention group with $<200$ ms responses excluded.

testing $(p=0.026)$. No changes in $\mathrm{JTT}_{\mathrm{R}}$ was observed in the sham group $(p=0.857)$.

The baseline SRTT curves are summarized in Figure 5C where a downward shift indicates improved reaction time. A negative correlation was observed between baseline reaction time and age ( $r=-0.488, p=0.016)$. There was a visible downward shift in SRTT curves for all groups. There was a significant learning effect from baseline to post-training (both $p<0.010$ ) and baseline to retention (both $p<0.009$ ) in the sham and tDCS groups. There was no significant learning effect in the HD-tDCS group. There was no decay in reaction time in any stimulation group.

\section{Replication}

The combined $\mathrm{PPT}_{\mathrm{L}}$ dataset for 3 days of training is shown in Figure 6. There was no difference in learning between either the sham groups $(p=0.402)$ or tDCS $(p=0.980)$ groups from both studies. For the combined data, a significant interaction effect of day and intervention group (rate of learning) was shown. Effect sizes were larger with sham participants placing $0.666 \pm 0.226$ more pegs each day compared to $1.04 \pm 0.375$ for tDCS and $1.00 \pm 0.320$ for HD-tDCS. The tDCS and HD-tDCS group outperformed the sham group in terms of the rate of pegs placed ( $\mathrm{tDCS} p=0.001, \mathrm{HD}-\mathrm{tDCS} p=0.012$ ). Effect sizes at the end of Day 3 were large for tDCS (Cohen's $d=1.265$ ) and HD-tDCS group (Cohen's $d=0.995$ ).

\section{Safety and Tolerability}

A total of 120 tDCS sessions were performed without any complications or serious adverse events. The most common reported sensation was itching (56\%) ranging from mild (75\%) to moderate $(25 \%)$ in severity. Itching was more common with tDCS $(78 \%)$ compared to sham $(48 \%, p=0.006)$ and HD-tDCS 


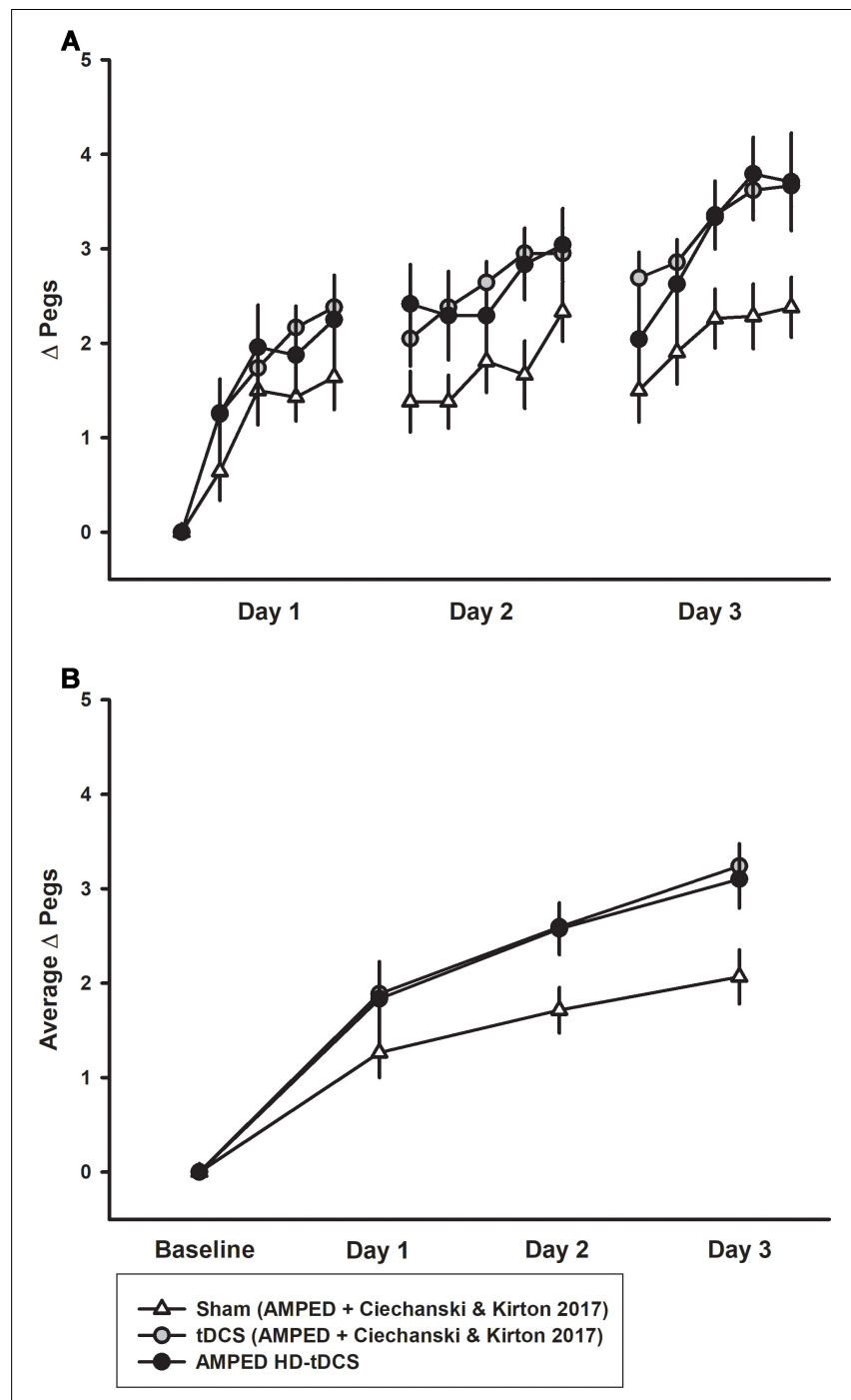

FIGURE 6 | Combined $P P T_{L}$ training data for sham and tDCS groups over 3 days. (A) Sham (white triangles, $n=14$ ) learning curves were inferior to both tDCS (gray circles, $n=14$ ) and HD-tDCS (black circles, $n=8$ ) groups.

(B) Mean daily learning for the same three groups form the combined studies. Both the tDCS and HD-tDCS groups placed more pegs each day as compared to sham.

(43\%, $p=0.001)$. Additional sensations included: unpleasant tingling 24\% (mild in 90\%) and burning 37\% (mild in 80\%), neither of which differed by treatment group. In the sham group, most sensations (90\%) lasted less than $2 \mathrm{~min}$. Sensations reported in tDCS and HD-tDCS persisted for the duration of the stimulation in $23 \%$ of $\mathrm{tDCS}$ and $3 \%$ of HD-tDCS sessions. One HD-tDCS participant reported a headache lasting less than 2 min. One tDCS participant felt mildly nauseated in one session. Tolerability rankings were comparable across groups: sham $3.8 \pm 1.1$, tDCS $4.1 \pm 1.0$, and HD-tDCS $3.9 \pm 1.2$, comparable to watching TV $(2.4 \pm 1.0)$ or a long car ride $(4.9 \pm 1.2)$. Participants were unable to correctly predict their treatment group. Baseline cognitive performance in all three domains was comparable across groups (Table 2, all $p>0.70$ ). No changes in cognitive function were found for any group (all $p>0.07$ ) with the single largest drop being in visual memory for the sham group.

\section{DISCUSSION}

Our findings suggest that application of both conventional and HD-tDCS is feasible, safe, and well-tolerated children. The addition of tDCS or HD-tDCS of the contralateral M1 can enhance motor learning compared to training alone. Children with lower performance at baseline may be more responsive to the effects of tDCS. Skill enhancement may spill over to untrained tasks and the untrained hand.

Improvement in motor learning with tDCS has been well established in adults during both single and multi-day sessions (Reis et al., 2009; Schambra et al., 2011; Prichard et al., 2014). Despite these promising findings, pediatric studies are limited. Our findings suggest that conventional and HD-tDCS may enhance motor skill learning with retained effects. Previous studies in adults suggested that motor learning occurs mainly through online effects but tDCS enhancement acted more selectively on offline consolidation (Reis et al., 2009). Limited work in pediatric populations suggests that tDCS may enhance motor learning by modulating online systems (Ciechanski and Kirton, 2016). Our findings here may occupy a middle ground between these bodies of evidence whereby some degree of offline effect was suggested for tDCS and possibly HD-tDCS where between session decay appeared to be less pronounced as compared to sham. Extension of previous adult studies demonstrating effects of tDCS administered after training (Tecchio et al., 2010) have not been replicated in children and represent a potential avenue to further elucidate potential mechanisms.

TABLE 2 | Group mean performance on the CNS Vital Signs neurocognitive battery.

\begin{tabular}{lccc}
\hline $\begin{array}{l}\text { Neurocognitive } \\
\text { domain }\end{array}$ & \multicolumn{3}{c}{ Participant group } \\
\cline { 2 - 4 } Time point & Sham & tDCS & HD-tDCS \\
\hline Visual memory & & \\
Baseline & $65.4(20.0)[32-92]$ & $67.4(26.6)[18-92]$ & $67.9(25.2)[25-96]$ \\
Post & $69.1(19.9)[37-92]$ & $75.0(20.7)[45-96]$ & $59.6(24.7)[25-95]$ \\
Follow-up & $43.5(27.0)[7-92]$ & $61.4(26.3)[10-97]$ & $65.3(28.0)[14-88]$ \\
Reaction time & & & \\
Baseline & $61.6(35.0)[2-95]$ & $53.9(36.5)[3-95]$ & $72.0(17.4)[37-87]$ \\
Post & $78.4(12.3)[53-96]$ & $61.3(26.6)[30-96]$ & $80.4(18.9)[45-98]$ \\
Follow-up & $68.1(26.6)[10-96]$ & $59.4(34.1)[12-90]$ & $66.4(25.0)[14-94]$ \\
Simple attention & & & \\
Baseline & $62.4(19.0)[23-79]$ & $60.4(27.6)[13-79]$ & $69.4(11.1)[50-79]$ \\
Post & $60.8(19.5)[23-79]$ & $57.5(32.6)[2-79]$ & $60.9(24.2)[13-79]$ \\
Follow-up & $56.4(26.1)[1-79]$ & $49.8(32.0)[1-79]$ & $73.4(11.0)[50-79]$ \\
\hline
\end{tabular}

Values are mean percentiles with (SD) and [range], higher values indicate better performance. tDCS, conventional anodal tDCS; HD-tDCS, high-definition tDCS. 
Mechanisms of tDCS are difficult to study in humans with even more limited knowledge in the developing brain (Antal et al., 2003; Anguera et al., 2007; Hummel et al., 2009; Lee et al., 2010b; Biabani et al., 2018). Anodal tDCS may modulate neuronal excitability leading to increased spontaneous neuronal firing rates. Such LTP-like enhancement and strengthening of neuronal activity between stimulated and distal locations may be similar to natural motor learning processes. Human studies suggest that tDCS paired with motor training may improve the efficacy of synaptic connections with lasting effects on cortical networks (Fritsch et al., 2010). GABA systems are likely crucial in motor learning (Stagg and Nitsche, 2011) and anodal tDCS may modulate M1 GABA in adults (Stagg et al., 2011). The use of advanced imaging before and after such trials may shed further light on the mechanisms of tDCS enhanced motor learning in children.

Motor learning and stimulation effects were not limited to the trained hand. Both pediatric and adult studies have shown tDCS-related improvements in the untrained hand (Pereira et al., 2011; Ciechanski and Kirton, 2016; Hamoudi et al., 2018). We have previously shown that M1 tDCS is associated with improvements in the untrained hand and spillover to untrained tasks (Ciechanski and Kirton, 2016). This transfer of skill was only evident in the active stimulation groups even though assessments were performed hours after stimulation when changes in neuronal excitability may still be present (Nitsche and Paulus, 2001; Moliadze et al., 2015). Improvements of the untrained hand could be secondary to various mechanisms (Anguera et al., 2007; Lee et al., 2010b). Increases in motor cortical excitability (Nitsche and Paulus, 2000), facilitation of motor performance (Antal et al., 2003; Nitsche et al., 2003; Boggio et al., 2006; Hummel et al., 2009), and potentiation of the formation of motor memories (Galea et al., 2010) have been reported after M1 tDCS. The "callosal access" and "bilateral access" hypothesis proposes that practice-induced motor engrams developed in the dominant hemisphere may be accessed in homologous regions of the opposite motor cortex (Anguera et al., 2007; Lee et al., 2010a). Others suggest the improvements reflect an increase of excitatory (or decrease of inhibitory) drive toward M1 (Biabani et al., 2018) and paired-pulse TMS studies have demonstrated suppression of intracortical inhibitory systems after tDCS (Biabani et al., 2018). MR spectroscopy studies have also shown a decrease in GABA after M1 tDCS (Stagg et al., 2014). Increases in BDNF have also been hypothesized to modulate neuroplastic potential (Biabani et al., 2018). Another possible theory invokes a role of mirror visual feedback affecting M1 plasticity (Nojima et al., 2012; von Rein et al., 2015). Mechanisms clearly remain to be defined.

The development of computational current models assists in understanding brain electric field strengths as well as potential differences in tDCS of children (Datta et al., 2009; Kessler et al., 2013). Age-related differences may include tissue structure, age-dependent differences in skull thickness, myelination, and volumes of CSF, gray matter, and white matter (Brain Development Cooperative Group, 2012). Pediatric current modeling suggests that electrodes placed on M1 produce diffuse cortical effects including contralateral M1 and bilateral premotor and supplementary motor areas (Kessler et al., 2013; Ciechanski et al., 2018). In contrast, HD-tDCS produces more focal stimulation with peak electric fields approximating functional cortical targets directly under the active electrode (Datta et al., 2009). Despite this potentially increased specificity, there have been few studies of HD-tDCS in motor learning and none in children. Improvement in motor behaviors in single (Doppelmayr et al., 2016) and multiday (Pixa et al., 2017) HDtDCS training studies appear consistent with our findings here. Our direct comparison of conventional to HD-tDCS provides further insight, though implications for mechanism remain speculative. One simple interpretation would be that stimulation of $\mathrm{M} 1$ is most important for enhancing motor learning effects as both montages accomplished this, likely to a comparable degree. A different hypothesis was that the stimulation of larger areas of the motor network (e.g., premotor and supplementary motor areas) and other frontal regions (prefrontal cortex) might be mediating the previously described effect of $1 \times 1$ tDCS. If this was the case, we would have expected the tDCS group to outperform the HD-tDCS group. That this did not occur provides indirect but informative evidence suggesting that $\mathrm{M} 1$ remains a major target for motor system neuromodulation approaches. We cannot rule out, however, that these other motor regions, or other areas such as primary sensory cortex, were not involved in the tDCS effects observed here.

Our study adds novel safety data. tDCS has been applied to thousands of subjects across a diverse array of brain disorders without evidence of harm (Bikson et al., 2016). Given that the wide distribution of conventional tDCS-induced electric fields and no previous HD-tDCS studies in children, we conducted screening cognitive tests before and after intervention with no signs of change. This supports the standing premise of functional targeting; neurostimulation likely only modulates neurophysiological processes that are themselves undergoing endogenous plastic change. In our population, tDCS was well-tolerated with itching and tingling being common as reported previously (Ciechanski and Kirton, 2016). HD-tDCS had comparable sensations and tolerability to tDCS. Adjusting saline concentration may be a method to improve tolerability and decrease sensation severity in children (Dundas et al., 2007, p. 200). Increasing the separation distance between stimulation electrodes may improve the tolerability of increased scalp current with HD-tDCS (Datta et al., 2009). The inability of subjects to guess their treatment assignment supports effective blinding of accepted sham techniques.

The translational significance of our findings is evident for the 17 million people in the world living with CP, the leading cause of lifelong disability (Oskoui et al., 2013). With M1 as the primary target, four early phase clinical trials of non-invasive stimulation paired with intensive motor therapy have shown evidence of safety and possibly efficacy in children with hemiparetic CP (Gillick et al., 2016, 2018; Kirton et al., 2016, 2017). That lower baseline skill level was also predictive of responding to stimulation-enhanced motor learning here is consistent with previous results in young adults (Ciechanski et al., 2017) and also therapeutically relevant if in fact those with poor motor 
skills may be more responsive. Clinical trials of neurostimulation in disabled pediatric populations require special considerations (Kirton, 2016) but should be driven by the first principles established in healthy populations as presented here.

Our study was limited by our modest sample size which was evidence-based but we encountered higher variability in outcomes than anticipated. This may have decreased our ability to fully define any potential differences between tDCS and HD-tDCS. Other factors may dictate differences in motor learning and response to neurostimulation including genetics (e.g., BDNF), gender, age, past experience, and environmental influences (Fritsch et al., 2010). Fatigue is another such factor and our study protocol was intense for young participants. We took extensive measures to ensure consistency with multiple breaks but cannot exclude fatigue effects.

\section{CONCLUSION}

tDCS and HD-tDCS of the primary motor cortex can enhance motor learning in healthy children over days with lasting effects. Trials of existing and emerging neurostimulation approaches are safe, feasible, and required to define and expand therapeutic potential for disabled children.

\section{REFERENCES}

Alam, M., Bikson, M., and Truong, D. (2014). Spatial and polarity precision of high-definition transcranial direct current stimulation (HD-tDCS). Brain Stimulat. 7:e11. doi: 10.1016/j.brs.2014.01.039

Ambrus, G. G., Al-Moyed, H., Chaieb, L., Sarp, L., Antal, A., and Paulus, W. (2012). The fade-in - Short stimulation - Fade out approach to sham tDCS - Reliable at $1 \mathrm{~mA}$ for naïve and experienced subjects, but not investigators. Brain Stimulat. 5, 499-504. doi: 10.1016/j.brs.2011.12.001

Anguera, J. A., Russell, C. A., Noll, D. C., and Seidler, R. D. (2007). Neural correlates associated with intermanual transfer of sensorimotor adaptation. Brain Res. 1185, 136-151. doi: 10.1016/j.brainres.2007.09.088

Antal, A., Kincses, T. Z., Nitsche, M. A., and Paulus, W. (2003). Manipulation of phosphene thresholds by transcranial direct current stimulation in man. Exp. Brain Res. 150, 375-378. doi: 10.1007/s00221-003-1459-8

Biabani, M., Aminitehrani, M., Zoghi, M., Farrell, M., Egan, G., and Jaberzadeh, S. (2018). The effects of transcranial direct current stimulation on short-interval intracortical inhibition and intracortical facilitation: a systematic review and meta-analysis. Rev. Neurosci. 29, 99-114. doi: 10.1515/revneuro-2017-0023

Bikson, M., Grossman, P., Thomas, C., Zannou, A. L., Jiang, J., Adnan, T., et al. (2016). Safety of transcranial direct current stimulation: evidence based update 2016. Brain Stimulat. 9, 641-661. doi: 10.1016/j.brs.2016.06.004

Bindman, L. J., Lippold, O. C., and Redfearn, J. W. (1964). The action of brief polarizing currents on the cerebral cortex of the rat (1) during current flow and (2) in the production of long-lasting after-effects. J. Physiol. 172, 369-382. doi: 10.1113/jphysiol.1964.sp007425

Boggio, P. S., Castro, L. O., Savagim, E. A., Braite, R., Cruz, V. C., Rocha, R. R., et al. (2006). Enhancement of non-dominant hand motor function by anodal transcranial direct current stimulation. Neurosci. Lett. 404, 232-236. doi: 10. 1016/j.neulet.2006.05.051

Brain Development Cooperative Group. (2012). Total and regional brain volumes in a population-based normative sample from 4 to 18 years: the NIH MRI study of normal brain development. Cereb. Cortex 22, 1-12. doi: 10.1093/cercor/ bhr018

Caparelli-Daquer, E. M., Zimmermann, T. J., Mooshagian, E., Parra, L. C., Rice, J. K., Datta, A., et al. (2012). A pilot study on effects of $4 \times 1$ high-definition

\section{DATA AVAILABILITY}

All relevant data generated and analyzed for this study is included in the manuscript.

\section{AUTHOR CONTRIBUTIONS}

LC, AG, PC, and AK conceived study design. LC and AG performed participant recruitment. LC, AG, PC, EZ, and $\mathrm{H}-\mathrm{CK}$ performed data collection. LC, AG, PC, and HC performed data analysis and drafting of the manuscript. LC, AG, PC, HC, H-CK, $\mathrm{EZ}$, and $\mathrm{AK}$ revised the manuscript. AK obtained funding.

\section{FUNDING}

This study was supported by the Canadian Institutes of Health Research (FDN-143294).

\section{ACKNOWLEDGMENTS}

The expert statistical support of Dr. Alberto Nettel-Aguirre is gratefully acknowledged.

tDCS on motor cortex excitability. Conf. Proc. IEEE Eng. Med. Biol. Soc. 2012, 735-738. doi: 10.1109/EMBC.2012.6346036

Ciechanski, P., Carlson, H. L., Yu, S. S., and Kirton, A. (2018). Modeling transcranial direct-current stimulation-induced electric fields in children and adults. Front. Hum. Neurosci. 12:268. doi: 10.3389/fnhum.2018.00268

Ciechanski, P., Cheng, A., Lopushinsky, S., Hecker, K., Gan, L. S., Lang, S., et al. (2017). Effects of transcranial direct-current stimulation on neurosurgical skill acquisition: a randomized controlled trial. World Neurosurg. 108, 876.e4884.e4. doi: 10.1016/j.wneu.2017.08.123

Ciechanski, P., and Kirton, A. (2016). Transcranial direct-current stimulation can enhance motor learning in children. Cereb. Cortex 27, 2758-2767. doi: 10.1093/ cercor/bhw114

Datta, A., Bansal, V., Diaz, J., Patel, J., Reato, D., and Bikson, M. (2009). Gyri precise head model of transcranial DC stimulation: Improved spatial focality using a ring electrode versus conventional rectangular pad. Brain Stimulat. 2, 201-207. doi: 10.1016/j.brs.2009.03.005

Dayan, E., Censor, N., Buch, E. R., Sandrini, M., and Cohen, L. G. (2013). Noninvasive brain stimulation: from physiology to network dynamics and back. Nat. Neurosci. 16, 838-844. doi: 10.1038/nn.3422

Dmochowski, J. P., Datta, A., Bikson, M., Su, Y., and Parra, L. C. (2011). Optimized multi-electrode stimulation increases focality and intensity at target. J. Neural Eng. 8:046011. doi: 10.1088/1741-2560/8/4/046011

Doppelmayr, M., Pixa, N. H., and Steinberg, F. (2016). Cerebellar, but not motor or parietal, high-density anodal transcranial direct current stimulation facilitates motor adaptation. J. Int. Neuropsychol. Soc. 22, 928-936. doi: 10.1017/ S1355617716000345

Dundas, J. E., Thickbroom, G. W., and Mastaglia, F. L. (2007). Perception of comfort during transcranial DC stimulation: effect of $\mathrm{NaCl}$ solution concentration applied to sponge electrodes. Clin. Neurophysiol. 118, 1166-1170. doi: 10.1016/j.clinph.2007.01.010

Elizabeth Reedman, S., Beagley, S., Sakzewski, L., and Boyd, R. N. (2015). The Jebsen taylor test of hand function: a pilot test-retest reliability study in typically developing children. Phys. Occup. Ther. Pediatr. 36, 292-304. doi: 10.3109/ 01942638.2015.1040576

Elsner, B., Kwakkel, G., Kugler, J., and Mehrholz, J. (2017). Transcranial direct current stimulation (tDCS) for improving capacity in activities and arm 
function after stroke: a network meta-analysis of randomised controlled trials. J. Neuroeng. Rehabil. 14:95. doi: 10.1186/s12984-017-0301-7

Fregni, F., and Pascual-Leone, A. (2007). Technology insight: noninvasive brain stimulation in neurology-perspectives on the therapeutic potential of rTMS and tDCS. Nat. Clin. Pract. Neurol. 3, 383-393. doi: 10.1038/ ncpneuro0530

Fritsch, B., Reis, J., Martinowich, K., Schambra, H. M., Ji, Y., Cohen, L. G., et al. (2010). Direct current stimulation promotes BDNF-dependent synaptic plasticity: potential implications for motor learning. Neuron 66, 198-204. doi: 10.1016/j.neuron.2010.03.035

Galea, M. P., Hammar, I., Nilsson, E., and Jankowska, E. (2010). Bilateral postsynaptic actions of pyramidal tract and reticulospinal neurons on feline erector spinae motoneurons. J. Neurosci. 30, 858-869. doi: 10.1523/ JNEUROSCI.4859-09.2010

Gardner, R. A., and Broman, M. (1979). The purdue pegboard: normative data on 1334 school children. J. Clin. Child Psychol. 8, 156-162. doi: 10.1080/ 15374417909532912

Garvey, M. A., Kaczynski, K. J., Becker, D. A., and Bartko, J. J. (2001). Subjective reactions of children to single-pulse transcranial magnetic stimulation. J. Child Neurol. 16, 891-894. doi: 10.1177/088307380101601205

Gillick, B., Rich, T., Nemanich, S., Chen, C.-Y., Menk, J., Mueller, B., et al. (2018). Transcranial direct current stimulation and constraint-induced therapy in cerebral palsy: a randomized, blinded, sham-controlled clinical trial. Eur. J. Paediatr. Neurol. 22, 358-368. doi: 10.1016/j.ejpn.2018.02.001

Gillick, B. T., Friel, K. M., Menk, J., and Rudser, K. (2016). "Therapeutic brain stimulation trials in children with cerebral palsy," in Pediatric Brain Stimulation: Mapping and Modulating the Developing Brain, eds A. Kirton and D. L. Gilbert (Amsterdam: Elsevier), 209-236.

Gualtieri, C. T., and Johnson, L. G. (2006). Reliability and validity of a computerized neurocognitive test battery, CNS vital signs. Arch. Clin. Neuropsychol. 21, 623-643. doi: 10.1016/j.acn.2006.05.007

Hamoudi, M., Schambra, H. M., Fritsch, B., Schoechlin-Marx, A., Weiller, C., Cohen, L. G., et al. (2018). Transcranial direct current stimulation enhances motor skill learning but not generalization in chronic stroke. Neurorehabil. Neural Repair. 32, 295-308. doi: 10.1177/1545968318769164

Honda, M., Deiber, M. P., Ibáñez, V., Pascual-Leone, A., Zhuang, P., and Hallett, M. (1998). Dynamic cortical involvement in implicit and explicit motor sequence learning. A pet study. Brain J. Neurol. 121( Pt 11), 2159-2173. doi: 10.1093/ brain/121.11.2159

Hummel, F. C., Heise, K., Celnik, P., Floel, A., Gerloff, C., and Cohen, L. G. (2009). Facilitating skilled right hand motor function in older subjects by anodal polarization over the left primary motor cortex. Neurobiol. Aging 31, 2160-2168. doi: 10.1016/j.neurobiolaging.2008.12.008

Kang, N., Summers, J. J., and Cauraugh, J. H. (2016). Transcranial direct current stimulation facilitates motor learning post-stroke: a systematic review and meta-analysis. J. Neurol. Neurosurg. Psychiatry 87, 345-355. doi: 10.1136/jnnp2015-311242

Kessler, S. K., Minhas, P., Woods, A. J., Rosen, A., Gorman, C., and Bikson, M. (2013). Dosage considerations for transcranial direct current stimulation in children: a computational modeling study. PLoS One 8:e76112. doi: 10.1371/ journal.pone.0076112

Kirton, A. (2016). Advancing non-invasive neuromodulation clinical trials in children: Lessons from perinatal stroke. Eur. J. Paediatr. Neurol. 21, 75-103. doi: 10.1016/j.ejpn.2016.07.002

Kirton, A., Andersen, J., Herrero, M., Nettel-Aguirre, A., Carsolio, L., Damji, O., et al. (2016). Brain stimulation and constraint for perinatal stroke hemiparesis: the plastic champs trial. Neurology 86, 1659-1667. doi: 10.1212/WNL. 0000000000002646

Kirton, A., Ciechanski, P., Zewdie, E., Andersen, J., Nettel-Aguirre, A., Carlson, H., et al. (2017). Transcranial direct current stimulation for children with perinatal stroke and hemiparesis. Neurology 88, 259-267. doi: 10.1212/WNL. 0000000000003518

Kuo, H.-I., Bikson, M., Datta, A., Minhas, P., Paulus, W., Kuo, M.-F., et al. (2013). Comparing cortical plasticity induced by conventional and high-definition $4 \times 1$ Ring tDCS: a neurophysiological study. Brain Stimulat. 6, 644-648. doi: $10.1016 /$ j.brs.2012.09.010

Lee, H. J., Lim, B. C., Hwang, H., Hong, J. S., Kim, E. K., Kim, H. S., et al. (2010a). Clinical presentations and neurodevelopmental outcomes of perinatal stroke in preterm and term neonates: a case series. J. Korean Med. Sci. 25, 888-894. doi: $10.3346 / \mathrm{jkms} .2010 .25 .6 .888$

Lee, M., Hinder, M. R., Gandevia, S. C., and Carroll, T. J. (2010b). The ipsilateral motor cortex contributes to cross-limb transfer of performance gains after ballistic motor practice. J. Physiol. 588, 201-212. doi: 10.1113/jphysiol.2009. 183855

Moliadze, V., Schmanke, T., Andreas, S., Lyzhko, E., Freitag, C. M., and Siniatchkin, M. (2015). Stimulation intensities of transcranial direct current stimulation have to be adjusted in children and adolescents. Clin. Neurophysiol. 126, 1392-1399. doi: 10.1016/j.clinph.2014.10.142

Nissen, M. J., and Bullemer, P. (1987). Attentional requirements of learning: evidence from performance measures. Cogn. Psychol. 19, 1-32. doi: 10.1016/ 0010-0285(87)90002-8

Nitsche, M. A., and Paulus, W. (2000). Excitability changes induced in the human motor cortex by weak transcranial direct current stimulation. J. Physiol. $527(\mathrm{Pt}$ 3), 633-639. doi: 10.1111/j.1469-7793.2000.t01-1-00633.x

Nitsche, M. A., and Paulus, W. (2001). Sustained excitability elevations induced by transcranial DC motor cortex stimulation in humans. Neurology 57, 1899-1901. doi: 10.1212/WNL.57.10.1899

Nitsche, M. A., Schauenburg, A., Lang, N., Liebetanz, D., Exner, C., Paulus, W., et al. (2003). Facilitation of implicit motor learning by weak transcranial direct current stimulation of the primary motor cortex in the human. J. Cogn. Neurosci. 15, 619-626. doi: 10.1162/089892903321662994

Nojima, I., Mima, T., Koganemaru, S., Thabit, M. N., Fukuyama, H., and Kawamata, T. (2012). Human motor plasticity induced by mirror visual feedback. J. Neurosci. 32, 1293-1300. doi: 10.1523/JNEUROSCI.5364-11.2012

Oskoui, M., Coutinho, F., Dykeman, J., Jetté, N., and Pringsheim, T. (2013). An update on the prevalence of cerebral palsy: a systematic review and metaanalysis. Dev. Med. Child Neurol. 55, 509-519. doi: 10.1111/dmcn.12080

Pereira, E. A. H., Raja, K., and Gangavalli, R. (2011). Effect of training on interlimb transfer of dexterity skills in healthy adults. Am. J. Phys. Med. Rehabil. 90, 25-34. doi: 10.1097/PHM.0b013e3181fc7f6f

Pixa, N. H., Steinberg, F., and Doppelmayr, M. (2017). High-definition transcranial direct current stimulation to both primary motor cortices improves unimanual and bimanual dexterity. Neurosci. Lett. 643, 84-88. doi: 10.1016/j.neulet.2017. 02.033

Prichard, G., Weiller, C., Fritsch, B., and Reis, J. (2014). Effects of different electrical brain stimulation protocols on subcomponents of motor skill learning. Brain Stimulat. 7, 532-540. doi: 10.1016/j.brs.2014.04.005

Reis, J., and Fritsch, B. (2011). Modulation of motor performance and motor learning by transcranial direct current stimulation: Curr. Opin. Neurol. 24, 590-596. doi: 10.1097/WCO.0b013e32834c3db0

Reis, J., Robertson, E. M., Krakauer, J. W., Rothwell, J., Marshall, L., Gerloff, C., et al. (2008). Consensus: can transcranial direct current stimulation and transcranial magnetic stimulation enhance motor learning and memory formation? Brain Stimul. 1, 363-369. doi: 10.1016/j.brs.2008.08.001

Reis, J., Schambra, H. M., Cohen, L. G., Buch, E. R., Fritsch, B., Zarahn, E., et al. (2009). Noninvasive cortical stimulation enhances motor skill acquisition over multiple days through an effect on consolidation. Proc. Natl. Acad. Sci. U.S.A. 106, 1590-1595. doi: 10.1073/pnas.0805413106

Richardson, J., Datta, A., Dmochowski, J., Parra, L. C., and Fridriksson, J. (2015). Feasibility of using high-definition transcranial direct current stimulation (HD-tDCS) to enhance treatment outcomes in persons with aphasia. NeuroRehabilitation 36, 115-126. doi: 10.3233/NRE-141199

Schambra, H. M., Abe, M., Luckenbaugh, D. A., Reis, J., Krakauer, J. W., and Cohen, L. G. (2011). Probing for hemispheric specialization for motor skill learning: a transcranial direct current stimulation study. J. Neurophysiol. 106, 652-661. doi: 10.1152/jn.00210.2011

Stagg, C. J., Bachtiar, V., Amadi, U., Gudberg, C. A., Ilie, A. S., Sampaio-Baptista, C., et al. (2014). Local GABA concentration is related to network-level resting functional connectivity. eLife 3:e01465. doi: 10.7554/eLife.01465

Stagg, C. J., Bachtiar, V., and Johansen-Berg, H. (2011). The role of GABA in human motor learning. Curr. Biol. 21, 480-484. doi: 10.1016/j.cub.2011.01.069

Stagg, C. J., and Nitsche, M. A. (2011). Physiological basis of transcranial direct current stimulation. Neuroscientist 17, 37-53. doi: 10.1177/1073858410386614

Tecchio, F., Zappasodi, F., Assenza, G., Tombini, M., Vollaro, S., Barbati, G., et al. (2010). Anodal transcranial direct current stimulation enhances procedural consolidation. J. Neurophysiol. 104, 1134-1140. doi: 10.1152/jn.00661.2009 
Tiffin, J., and Asher, E. J. (1948). The Purdue pegboard; norms and studies of reliability and validity. J. Appl. Psychol. 32, 234-247. doi: 10.1037/ h0061266

Villamar, M. F., Volz, M. S., Bikson, M., Datta, A., Dasilva, A. F., and Fregni, F. (2013). Technique and considerations in the use of $4 \times 1$ ring high-definition transcranial direct current stimulation (HD-tDCS). J. Vis. Exp. 77:e50309. doi: $10.3791 / 50309$

Vines, B. W., Nair, D. G., and Schlaug, G. (2006). Contralateral and ipsilateral motor effects after transcranial direct current stimulation. Neuroreport 17, 671-674. doi: 10.1097/00001756-200604240-00023

von Rein, E., Hoff, M., Kaminski, E., Sehm, B., Steele, C. J., Villringer, A., et al. (2015). Improving motor performance without training: the effect of combining mirror visual feedback with transcranial direct current stimulation. J. Neurophysiol. 113, 2383-2389. doi: 10.1152/jn.00832. 2014
Zewdie, E., and Kirton, A. (2016). "TMS basics: single and paired pulse neurophysiology," in Pediatric Brain Stimulation: Mapping and Modulating the Developing Brain, eds A. Kirton and D. L. Gilbert (Amsterdam: Elsevier).

Conflict of Interest Statement: The authors declare that the research was conducted in the absence of any commercial or financial relationships that could be construed as a potential conflict of interest.

Copyright (C) 2018 Cole, Giuffre, Ciechanski, Carlson, Zewdie, Kuo and Kirton. This is an open-access article distributed under the terms of the Creative Commons Attribution License (CC BY). The use, distribution or reproduction in other forums is permitted, provided the original author(s) and the copyright owner(s) are credited and that the original publication in this journal is cited, in accordance with accepted academic practice. No use, distribution or reproduction is permitted which does not comply with these terms. 